\title{
A Report on the 3-D Acoustic Working Group Meeting at Long Beach, MS, July 7-8, 1988
}

\author{
by \\ James F. Lynch \\ Woods Hole Oceanographic Institution \\ Woods Hole, Massachusetts 02543 \\ and \\ Ching-Sang Chiu \\ Institute for Naval Oceanography, Stennis Space Center, \\ MS 39529-5005
}

June 1989

\section{Technical Report}

Funding was provided by the Office of Naval Research under contract Number N00014-88-K-0363.

Reproduction in whole or in part is permitted for any purpose of the

United States Government. This report should be cited as:

Woods Hole Oceanog. Inst. Tech. Rept., WHOI-89-16.

Approved for publication; distribution unlimited.

Approved for Distribution:

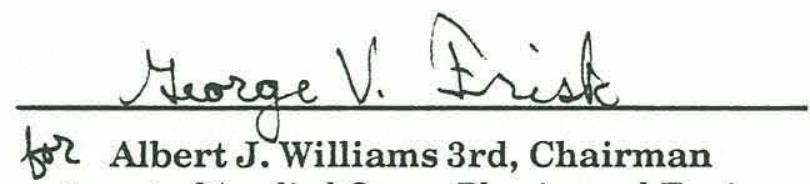

Department of Applied Ocean Physics and Engineering 


\title{
A report on \\ the $3-\mathrm{D}$ acoustic working group meeting at Long Beach, MS, July 7-8, 1988
}

\author{
James F. Lynch \\ Woods Hole Oceanographic Institution, Woods Hole, MA 02543 \\ Ching-Sang Chiu ${ }^{1}$
}

Institute for Naval Oceanography, Stennis Space Center, MS 39529-5005

\footnotetext{
${ }^{1}$ Naval Postgraduate School, Code 68Ci, Monterey, CA 93943
} 


\section{Contents}

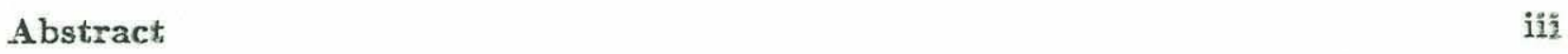

1 Introduction/Objectives 1

2 Summary of July 7 Presentations 3

2.1 Harrard Open Ocean Model by S. Glenn . . . . . . . . . . . . . . . 4

2.2 3-D Parabolic Equation Based Acoustic Model by D. Lee, W. Siegmann . . . . . . . 5

2.3 3-D Coupled Mode Acoustic Model by C.S. Chiu and 3-D PE Acoustic Wavefield in a Shallow Water Waveguide by M.D. Collins . . . . . . . . . . . 5

2.4 3-D Acoustic Ray Tracing with Realistic Ocean Input by J.F. Lynch and 3-D Acoustic Ray Tracing - Ocean Current Nonreciprocity Effects by J. Mercer . . . . . . . . 6

2.5 Improving on Acoustic Ray Methods - Gaussian Beams by H. Bucker . . . . . . 7

2.6 Stepwise Coupled Acoustic Modes by R. Evans, Improved Coupled Mode Acoustic Calculations by H. Uberall and Nx2D Adiabatic Modes by J. Perkins . . . . . . . 8

2.7 Vertical Modes and Horizontal Rays by H. Weinberg . . . . . . . . . . . . 9

2.8 Nx2D PE Acoustic Modeling - PESOGEN by F. Tappert . . . . . . . . . . . . 10

2.9 Finite Element Modeling by J. Murphy and S. Chin-Bing . . . . . . . . . . . 10

2.10 Intercomparison of Acoustic Models by J. Matthews . . . . . . . . . . . . . 11

2.11 The Calculational Frequency Method for Acoustic PE Models by M. Head, Parabolic Equation Marching Algorithms by J. McCoy and Bistatic Bottom Reverberation

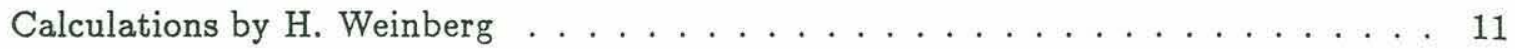

2.12 Full Wave Elastic Scattering Calculation (pseudospectral method) by T. Charette and (regular difference method) by M. Dougherty . . . . . . . . . . . 12 
3 Summary of issues discussed, findings and outlook

3.1 Ocean Model/Data/Acoustic Model Interface . . . . . . . . . . . . . . 13

3.2 Ocean Model Sensitivity . . . . . . . . . . . . . . . . . . . . . 14

3.3 Interpolation of Ocean Sound Velocity and Current Data . . . . . . . . . . . 14

3.4 Acoustic Model Sensitivity . . . . . . . . . . . . . . . . . . . . . 15

3.5 Miscellaneous . . . . . . . . . . . . . . . . . . . 16

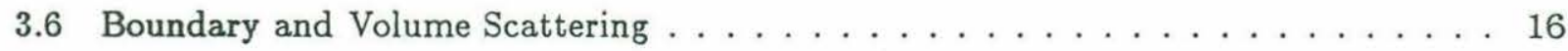

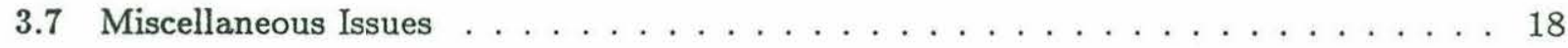

3.8 Concluding Remarks . . . . . . . . . . . . . . . . . . . . . . . 19

4 Acknowledgements $\quad 21$

5 Appendix A: List of Attendees $\quad 22$

6 Appendix B: List of Presentations 24 


\begin{abstract}
At the request of ONR Code $1125 \mathrm{OA}$, the Woods Hole Oceanographic Institution (Dr. James F. Lynch) convened a workshop to bring together a group of acoustic and ocean modelers to review and discuss
\end{abstract}

1. the state of development and the need for three-dimensional numerical acoustic research propagation and scattering models;

2. the interfacing of acoustic models with available oceanographic data and ocean model outputs.

The workshop was hosted by the Institute for Naval Oceanography (Dr. Ching-Sang Chiu) at Long Beach, MS on July 7-8, 1988. This report summarizes the research presentations and the recommendations made by the group. The workshop was an initial attempt to promote the interaction between the ocean and acoustic modeling communities. This interaction between the communities is essential to the development of truly interactive basic research acoustic and ocean models. We anticipate more workshops of such nature to be held in the future. The findings and recommendations generated by these workshops are expected to have a strong impact on the direction of future three-dimensional modeling research in both acoustics and oceanography. 


\section{Introduction/Objectives}

The spatial and temporal sensitivity of the 3-D acoustic wavefield to spatial and temporal gradients in the sound velocity field is neither fully understood nor presently predictable on a quantitative basis. To increase our understanding of 3-D acoustic wavefield propagation and scatter in the heterogeneous ocean environment, the ocean acoustics community must develop a 3-D acoustic modeling capability which is integrated with physical oceanographic modeling efforts. To illustrate the need, consider a low frequency $(50-1000 \mathrm{~Hz})$ acoustic wavefield propagating over long distances $(\approx 500-1000$ km) through oceanographic eddy fields, frontal systems and associated internal gravity wavefields. Depending on the strength of their sound velocity fluctuations, these features can refract an acoustic wavefield in both the vertical and horizontal. In addition, acoustic wavefield interaction with the bottom/subbottom results in 3-D scatter, refraction, reflection, and diffraction of the incident acoustic wavefield. Interaction with the surface gravity wavefields results in 3-D scatter and reflection. These 3-D environmental effects cannot be modeled by existing 2-D or $\mathrm{N}$ by 2-D acoustic propagation and scatter models.

Research in 3-D acoustic and physical oceanographic modeling is in its infancy. Three dimensional acoustic propagation codes that are capable of processing gridded ocean model output data or oceanographic field data have only recently become available. The pioneering work of an acoustic modeling group (lead by Lee at NUSC and including Siegmann and Jacobson at RPI, St. Mary at University of Massachusetts, and Shultz at Yale) and an ocean modeling effort (lead by Robinson at Harvard) involves pairing NUSC's 3-D parabolic equation acoustic code, FOR3D, with the Harvard Open Ocean Model (HOOM). Recently, a 3-D coupled mode (Chiu, INO) and a 3-D ray code

(Lynch, WHOI) have been adapted to accept 3-D sound velocity fields calculated by the HOOM. 
The coupled mode code was implemented at INO, whereas the ray code is a WHOI upgrade of NOAA's Hamiltonian Acoustic Raytracing Program for the Ocean (HARPO).

At this point in time, there are many unresolved issues concerning the interfacing of ocean models or oceanographic field data and 3-D acoustic models. For example, the accuracy and horizontal and vertical resolution requirements on the 3-D sound speed fields input to the 3-D acoustic model have not been specified. Also, the evaluation and intercomparison of the accuracy of various 3-D acoustic models, each containing a different degree of completeness of physics and using different numerical algorithms, has not been addressed. Similar claims can be made in regard to existing 4-D numerical ocean models. To quantitatively define these issues and to expedite progress in the emerging field of 3-D ocean-acoustic model interfacing, the acoustic and ocean modelers must work together as a team. An important first step is to establish communications between the acoustic and ocean research communities.

In that spirit and at the request of the Office of Naval Research, Code 1125OA, the Woods Hole Oceanographic Institution (Dr. James F. Lynch) convened and the Institute for Naval Oceanography hosted a two-day (July $7-8,1988$ ) 3-D ocean-acoustic modeling working group meeting at the University of Southern Mississippi, Long Beach, MS. A list of attendees is provided in Appendix A. Nineteen presentations to review the status of current research in 3-D ocean and acoustic modeling (titles and speakers listed in Appendix B) were given on the first day. A brief summary of the material presented in each talk is given in Section 2. A discussion session, held on the second day, focused on the following issues: (1) the integration of 3-D ocean models and 3-D acoustic models (e.g., is standardization possible), (2) the input of 3-D sound velocity and water velocity field data (e.g., by objective analysis) into acoustic propagation models, (3) error analysis on the acoustic propagation models and the ocean models (i.e., develop time and accuracy benchmarks), 
and (4) the definition of the statistical and deterministic treatment of the modeled 3-D acoustic wavefield (e.g. what components of rough surface scattering and internal wave and fine structure scattering should be treated statistically). Section 3 of the report summarizes the findings and the recommendations of the discussion session.

To stimulate interaction between the ocean and acoustics 3-D modeling communities future meetings were and are planned. A special session on ocean-acoustic model interfacing was held at the second IMACS Symposium on Computational Acoustics (March 15-17, 1989; Princeton, NJ). Also, INO, which holds an annual colloquium on mesoscale ocean science and prediction, plans to invite acoustic modelers to attend the 1989 session next summer. It is hoped that future workshops of this nature will attract a broader cross section of ocean modeling community. The participation and interaction of the ocean modeling community with the acoustic modeling community is essential to assure that the hierarchical ocean models include the physics necessary to address the spatial and temporal scales which are relevant to the 3-D acoustic modeling community.

\section{Summary of July 7 Presentations}

The workshop (July 7) began with introductory remarks (M. Orr, Code 1125 OA, ONR) highlighting the meeting objectives. Nineteen research presentations which discussed the state of the art 3-D ocean prediction models and 3-D acoustics models followed. The speakers outlined the models' theoretical and numerical bases, strengths and weaknesses, current capabilities and potential improvements, speeds and accuracies, advantages and practical limits, input/output formats and applications. The presentations acquainted the ocean and acoustic workshop participants with each other's work and provided the background for the discussion sessions. 


\subsection{Harvard Open Ocean Model by S. Glenn}

Glenn (Harvard University) discussed the Harvard University Open Ocean Model (HOOM) and was followed by Lee, Lynch and Chiu who, respectively, outlined the three different 3-D acoustic numerical codes which are, at present, capable of accepting the HOOM 3-D gridded output sound velocity fields and calculating 3-D acoustic wavefields. Their work, in close collaboration and coordination with each other and with investigators at RPI (Siegmann and Jacobson), Yale (Shultz), University of Massachusetts (St. Mary) and Harvard University (Robinson and Glenn), constitute the beginning of the 6.1 research effort to interface 3-D ocean and acoustic models.

The Harvard University open ocean model is used to forecast the position of features, such as the Gulf Stream and associated eddies. Currently, this quasigeostrophic, open boundary, regional ocean model is configured to calculate the ocean streamline field for six vertical levels and a horizontal increment of $15 \mathrm{~km}$. Satellite infrared imagery, altimeter data, and AXBT data are used to estimate the locations of the walls of the Gulf Stream, the number and locations of cold and warm rings, as well as other input parameters, such as the diameter of and maximum current in each ring. Canonical fronts and rings derived from this data are used to initialize the HOOM model. The HOOM model then integrates forward in time to predict the ocean's evolution. The output products include the forecast streamfunction, temperature, current and sound speed at every computational mesh point. The latter two products are central to doing acoustic predictions. At this point the sound speed is estimated from archival data and not updated with AXBT data. In all likelihood it may not be representative of the existing sound velocity field. Sensitivity tests must be done to determine the estimated sound velocity field's accuracy. Although the HOOM model resolution is largely inadequate, both horizontally and vertically, for acoustic prediction purposes, Glenn pointed 
out that the resolution can be increased at the expense of computational time. It is therefore vital for the ocean and acoustic modelers to work together to define the optimal number and location of the vertical levels and the horizontal mesh interval required for acoustic wavefield prediction.

\subsection{3-D Parabolic Equation Based Acoustic Model by D. Lee, W. Siegmann}

Lee (NUSC) discussed the capabilities, advantages and limitations of a three-dimensional parabolic approximation acoustic code called FOR3D, co-developed by him, Botseas at NUSC, Siegmann and Jacobson at RPI, Schultz at Yale University, and St. Mary at University of Masschusetts. Inherent to the parabolic approximation is the neglect of the backscattered acoustic field and the invalidity of the solution near the source. These two limitations exist in most other acoustic models as well. Since Lee's finite difference solution is generated using a marching scheme which solves only two tridiagonal systems, the computation is economical. At present, FOR3D can handle long range, low frequency, wide angle calculations in a three-dimensionally varying shallow or deep water

environment. It also accepts arbitrary side walls, surface and bottom boundary conditions, and accounts for bottom and surface interactions. These investigators will continue to increase the capabilities of the code to make it a superior basic research tool. For instance, they plan to include a beamforming capability in the near future. Moreover, Lee and his collaborators will continue to improve the numerical algorithms to make the computation more efficient and physically acccurate.

\subsection{3-D Coupled Mode Acoustic Model by C.S. Chiu and 3-D PE Acoustic Wavefield in a Shallow Water Waveguide by M.D. Collins}

In separate presentations, Chiu (INO) compared the acoustic wavefield calculated from a 3-D coupled mode acoustic model to the wavefield from a $\mathrm{N}$ by 2-D coupled mode model for the case of 
long range propagation in a Gulf Stream forecast from the HOOM, and Collins compared the 3-D $\mathrm{PE}$ acoustic wavefield to the $\mathrm{N}$ by 2-D PE wavefield in a shallow water waveguide over a corrugated bottom. Both calculations were done at an acoustic frequency of $50 \mathrm{~Hz}$. They found significant differences between the 3-D and $\mathrm{N}$ by 2-D solutions. In Chiu's case, transmission losses differ by up to $5 \mathrm{db}$ and phases of a given mode differ by up to $90^{\circ}$ over a range of $400 \mathrm{~km}$. An error of such magnitude in the mode phases certainly has significant ramifications in phased array applications and acoustic tomography. Collin's (PE) study shows that the convergence zones given by the two solutions are completely out-of-phase in a range of a few kilometers. The results of these two investigators demonstrate that azimuth coupling or horizontal deflection of acoustic energy must be accounted for when computing propagation through strong boundary current systems or in shallow water environments with rough bottoms.

\subsection{3-D Acoustic Ray Tracing with Realistic Ocean Input by J.F. Lynch and 3-D Acoustic Ray Tracing - Ocean Current Nonreciprocity Effects by J. Mercer}

The Hamiltonian Acoustic Ray Tracing Program for the Ocean (HARPO) is a 3-D code developed during the past several years by Jones, Riley and Georges at NOAA/WPL. Due to the nature of the geometric approximation, this asymptotic method works well at high frequencies and for broadband calculations, but breaks down at low frequencies and at caustics. To simplify the numerical calculation, in particular, the ease of calculation of continuous first derivatives, HARPO

originally accepted only analytical canonical ocean features as input, e.g. straight line fronts and Gaussian eddies.

Lynch (WHOI) described his research work to upgrade HARPO to accept oceanographic objec- 
tive maps or ocean model output data, to include 3-D intensity calculations for arbitrary bottom topography and upgrade its eigenray searching capabilities. Chiu and Lynch are assessing gridded ocean model output sound speed and ocean current profile data representation and interpolation techniques to determine their impact on the accuracy of acoustic wavefield calculation. Field representations are via empirical orthogonal functions (EOF) and quasigeostrophic (QG) ocean modes, which can greatly decrease the amount of computer storage needed for the fields.

HARPO can calculate the acoustic wavefield propagating in a medium having variable sound speeds and ocean currents and irregular interfaces. Since the code decomposes the acoustic field into rays, it is an ideal code for tomographic analyses and feasibility studies. Mercer (APL/UW) has simulated a basin scale reciprocal tomography experiment to study the effect of nonreciprocity of forward and reverse eigenrays when currents were present. For a model basin containing four eddies and a boundary jet, he found differences of up to $40 \%$ in the acoustic ray arrival times when compared to calculations which did not include currents. The difference was also found to be proportional to the number of eddies and to decrease linearly with decreasing current magnitude. Mercer pointed out that the 2-D and 3-D ray trace results were only slightly different in those cases considered by him. Lynch, however, plans to experimentally study the effects of horizontal refraction on acoustic tomography in less benign environments, for example, over rough topography in a shallower ocean, and in the Gulf Stream.

\subsection{Improving on Acoustic Ray Methods - Gaussian Beams by H. Bucker}

Singularities at caustics and discontinuities at shadow zones are two undesirable field artifacts generated in conventional acoustic ray tracing. Gaussian beam tracing, as discussed by Bucker (NOSC) is a method to eliminate these artifacts. The incorporation of the method seemingly 
requires only a simple modification to standard ray trace codes. A beam with a Gaussian intensity profile normal to each ray is assumed. In addition to performing the standard ray tracing, a pair of differential equations governing the corresponding beamwidth and curvature are integrated. The beam makes significant corrections at caustics and in shadow zones. Also, unlike an infinitely narrow ray, it is less sensitive to rough boundaries. These attractive improvements plus the elimination of the computationally expensive eigenray finding procedure in the acoustic intensity calculations make beam tracing a far more powerful method for the construction of solutions in the highfrequency regime than standard ray tracing.

\subsection{Stepwise Coupled Acoustic Modes by R. Evans, Improved Coupled Mode Acoustic Calculations by H. Uberall and Nx2D Adiabatic Modes by J. Perkins}

In a discretely range-dependent ocean waveguide, an exact far-field solution that contains both the forward and backscattered acoustic energy can be obtained using the stepwise coupled acoustic mode method of Evans (SYNTEC). In the first half of his presentation, Evans outlined the algorithm to exactly compute the 3-D solution for the case of propagation over a symmetric, staircase seamount. He proposed to use this stepwise coupled mode solution as a benchmark for other approximate 3-D numerical codes. In the second half of this presentation, Evans discussed the continuous representation of bottom roughness. He recommended the use of a polynomial interpolating function in each triangular facet in a triangulation of the horizontal plane.

A code for computing the acoustic field in a layered range-dependent environment has been under development in the Catholic University of America under Uberall for the past few years. The code is presently being modified to permit the modeling of absorption and shear effects in a 
layered ocean bottom, the azimuth coupling of acoustic energy (i.e., 3-D effects), and the inclusion of the Rutherford- Hawker sloping-boundary correction.

The uncoupled (or adiabatic) acoustic mode theory is an approximation to the coupled mode theory. It is valid for low acoustic frequencies and slowly varying environments. It is particularly useful for the computation of the acoustic wavefields near the SOFAR axis where the weakly coupled lower modes dominate. It is also computationally tractable for some deep ocean propagation problems. Perkins (NRL) presented some of the applications of the Wide Area Rapid Acoustic Prediction adiabatic $\mathrm{N}$ by 2-D mode code (WRAP) developed by him in conjunction with Kuperman and Porter at NRL. An application shown was the modeling of horizontal and vertical arrays responses in a North Atlantic eddy field. The model included the effects of ambient noise. Two major computational advantages of the adiabatic mode method, as pointed out by Perkins, are that (1) only a re-sum of the normal modes with different weights is required when the source and receiver locations change and (2) the mode functions in the lower ocean can be stored to avoid recalculations since they change negligibly in the region below the main thermocline. An interesting video movie, shown by Perkins, demonstrated the use of color graphics to clearly display and convey the complex interactions between the acoustic wavefield and ocean processes.

\subsection{Vertical Modes and Horizontal Rays by H. Weinberg}

Weinberg (SYNTEC) is reinvestigating the 3-D computational method of vertical modes and horizontal rays which he introduced fifteen years ago. At that time, his adiabatic mode method was the only algorithm that could perform coupled azimuth 3-D calculations. The method was not developed as it was computationally too intensive for the serial computers available at that time. The techniques may be suitable for recently introduced parallel processing computer systems. Weinberg 
pointed out that the computations of the vertical modes from location to location are independent, as are the computations of the horizontal rays launched at different angles.

\subsection{Nx2D PE Acoustic Modeling - PESOGEN by F. Tappert}

Tappert (Univ. Miami) has applied firmware parallel processing techniques to a $\mathrm{N}$ by 2-D acoustic propagation model. The resulting very wide angle $\mathrm{PE}$ code has the computational speed necessary for naval operational use. Tappert's PE model assumes uncoupled azimuth, thus allowing the computations in each angular sector to be done in parallel. The computation speed increases in proportion to the increase in the number of processors. Tappert argued that the uncoupled azimuth assumption is valid in most deep ocean environments. He emphasized that this $\mathrm{N}$ by 2-D PE model does to some extent account for horizontal refraction. Quantitative support for the argument was not provided.

\subsection{Finite Element Modeling by J. Murphy and S. Chin-Bing}

Murphy (Univ. of New Orleans) and Chin-Bing (NORDA) described a 2-D elastic wavefield finite element model which calculated both the forward and backscattered acoustic wavefield. This code, which can be expanded to 3-D, can provide an accurate description of the acoustic field near the source. The triangular elements can be stretched, compressed, and moved around to accommodate exact boundary conditions, and the nodes can be located wherever data are available. With future installation of a marching algorithm, Murphy expects the finite element method to be applicable to long range calculations. In each frame in the march, the solution will contain the backscattered energy coming from the neighboring frames only. In his talk, Murphy stressed that the limited memory of most research computers is a more detrimental problem to the use of finite element 
codes than computational slowness. His view was shared by other modelers as well.

\subsection{Intercomparison of Acoustic Models by J. Matthews}

King and Matthews (NORDA) have recently completed a project to select Navy standard range dependent (i.e., 2-D) acoustic propagation models. ASTRAL 2 was selected for speed whereas Parabolic Equation was chosen for accuracy. Matthews illustrated the complexities of model evaluation. These included careful evaluation, editing and formating of input data, as well as the reconfiguring of candidate models to accept common input and to produce a uniform output. He also indicated that models must be evaluated according to objectives and criteria established a priori, with priorities assigned to the various aspects of model predictions and the conditions under which the models will be operated. Matthews further urged the establishment of criteria for the evaluation of 3-D models at the inception of the 3-D modeling effort. These criteria have yet to be formulated for the 3-D acoustics case. Matthews' 2-D experiences should be utilized during the initial 3-D mode, ray, and PE code intercomparison studies being conducted by Chiu, Lynch, Siegmann and Lee. One of the criteria for the evaluation of 3-D models which was not addressed by Matthews' 2-D work was the number of data points needed to characterize a 3-D ocean medium.

\subsection{The Calculational Frequency Method for Acoustic PE Models by M. Head, Parabolic Equation Marching Algorithms by J. McCoy and Bistatic Bottom Reverberation Calculations by $\mathrm{H}$. Weinberg}

Head (NORDA) outlined the use of the calculation-frequency method (CFM) which efficiently calculates range-averaged high-frequency bottom surface losses. The CFM saves computational time by solving the parabolic equation for low frequency propagation, and then applying the volume 
attenuation and boundary loss calculation appropriate to higher frequencies. McCoy discussed a phase-space marching algorithm used to solve higher order parabolic ray equations. The split-step algorithm is a special case of the marching algorithm. He also introduced a technique to stabilize marching, which he referred to as phase-space filtering. The stabilization technique is equivalent to the elimination of plane waves propagating at high angles in the marched solution. Weinberg presented his analysis of a bistatic reverberation data set obtained with point explosive sources. He found that a broken mirror model for bottom scatter together with the introduction of coherence into the reverberation computation fit the data extremely well.

\subsection{Full Wave Elastic Scattering Calculation (pseudospectral method) by $\mathrm{T}$. Charette and (regular difference method) by M. Dougherty}

There were two talks on seismo-acoustics, one by Dougherty (WHOI), the other by Charette (MIT). They both model the compressional and shear wave fields in the crust and mantle by numerically solving the full elastic wave equation. They place their source slightly above the ocean floor. Such time domain full wave approaches are important as they are the only methods which can exactly solve the elastic (or anelastic) scattering problem for realistic ocean bottoms. Although the direct construction of a numerical solution to the wave equation is computationally expensive and thus limited to a short range and low frequency applications, it does produce a time-domain broadband result. Current research issues include the conversion of these models to $3-\mathrm{D}$ and embedding them in fast 3-D propagation codes which calculate the acoustic wavefield in the non-scattering regions. Charette used the psuedo-spectral numerical method which requires a spatial sampling of only two points per wavelength. Dougherty uses the regular difference methods which requires more (about 10) points per wavelength but handles sharp gradients and discontinuities in a superior fashion. 


\section{Summary of issues discussed, findings and outlook}

A summary of the discussion sessions held on July 8 follows.

\subsection{Ocean Model/Data/Acoustic Model Interface}

The interdisciplinary interaction of the ocean acoustic community and the oceanography community will permit the integration of ocean acoustics (whether 2-D or 3-D) and physical oceanographic models. It was evident from the meeting that, in general, acousticians and oceanographers are not familiar with each other's endeavors. If acousticians are to be intelligent users of ocean forecasting models, and are to influence the oceanographic community in developing models which are useful for acoustics work, some effort must be made to learn physical oceanography.

During this meeting, Glenn from Harvard and Mooers and Chiu from INO represented the ocean modeling community. Due to the lack of representation by other ocean modeling groups, the Harvard Open Ocean Model (HOOM) was the only model discussed in depth. Since the HOOM is at present the ocean forecasting model that is best adapted to real world data input, and calculating an output sound velocity profile for acoustic models, this was probably not a serious drawback. It

did, however, preclude ocean acoustician exposure to ocean models which include more complete physics than the HOOM.

Briefly, the HOOM is a quasigeostrophic, open boundary condition model that can assimilate observations (i.e. satellite IR and aircraft XBT) and forecast ocean feature time evolution. The quasigeostrophic approximation limits its use to slowly changing ocean features (ruling out, for instance, internal waves, etc.), whereas the open boundary conditions mean that one must specify the ocean current structure (actually vorticity) on the boundaries of the region modeled. For the 
Gulf Stream, where the inflow and outflow of that current dominate the boundary conditions, open boundaries can be reasonably specified. It should be noted that many groups (Harvard included) are working on so called "primitive equation" models with both open and non-open (coastal) boundary conditions. These models are closer approximations to the basic Navier-Stokes equation than the quasigeostrophic models. Such primitive equation models can handle shorter time scale and length scale processes.

\subsection{Ocean Model Sensitivity}

The temporal and spatial scales which ocean models can resolve was discussed. The Harvard Model resolves temporal scales with a one day resolution, has a $15 \mathrm{~km}$ horizontal grid spacing in $\mathrm{x}$ and $y$, and currently has six grid levels in the $\mathrm{z}$ direction. The horizontal, out of plane, deflections of acoustic rays calculated for the Gulf Stream by Lynch were a few kilometers over ranges of several hundred kilometers. These calculations gave rise to some concern over whether a $15 \mathrm{~km}$ horizontal grid used in the HOOM was adequate for acoustic interpolation purposes. The adequacy of such a grid size to incorporate the fine scale features of the Gulf Stream was also questioned. Siegmann noted that the six vertical levels used in the HOOM was inadequate and that Harvard was now working on nine levels, with the extra levels being most needed near the surface. It was felt that mesh spacing limitations were primarily due to limits in computer storage and could be addressed.

\subsection{Interpolation of Ocean Sound Velocity and Current Data}

The acoustics community interpolates the sound velocity and current fields, calculated from ocean models or derived from field measurements, in a number of ways. The quantitative effects of each interpolation scheme will have to be understood in order to determine if standardization is 
necessary. Some interesting ideas were discussed concerning the interpolation of the ocean sound speed and current profiles and bathymetry data. By using either empirical orthogonal functions or ocean quasigeostrophic modes, one might exploit the fact that the ocean has a red spectrum (i.e. may be represented by a few low order modes) and minimize the core storage needed to represent the sound speed and ocean current field. Moreover, one could use the Gauss-Markoff theorem ("objective analysis") to interpolate incomplete, gappy sound speed and/or current data. Aliasing phenomena will have to be addressed. Anisotropy in the bottom statistics and non-redness of the spectrum make objective analysis/model approaches less attractive for the interpolation of bathymetry data. Evans showed some work on the triangularization interpolation method in his talk. This is one alternative to spectral methods. (Both spectral and grid methods, as well as others are nicely discussed in the Book "Spatial Statistics" by Brain Ripley, J. Wiley, 1981.)

\subsection{Acoustic Model Sensitivity}

The precision of the input sound velocity and ocean current fields needed by a research 3-D acoustic propagation model are dependent upon the research application. For instance, tomographic inversions require "background" models which reasonably represent the initial sound velocity profiles. If one is interested in the effect of fine-scale processes on acoustic propagation, these also must obviously be modeled. And although source localization schemes such as beamforming and matched field processing tend more toward 6.2 and 6.3 research, in the interest of transitioning the 6.1 work, signal processing needs should be kept in mind. For instance, ocean model accuracy needs for conventional beamforming are much less stringent than those for matched field. The magnitude of error bars associated with the ocean model outputs was discussed. It seems that these can be generated by analyzing the errors in the ocean model input data, and iteratively running 
the ocean models. This is not a time effective procedure and has not been implemented. Neither the acoustic or the oceanography modeling communities could quantitatively address the issue of model accuracy at this point in time.

\subsection{Miscellaneous}

Arctic ice and ocean mixed layer models were discussed. By Arctic "ocean ice models" we mean thermodynamic models (e.g., the Hibler model) which are used to predict ice cover, ice thickness, etc. and the thermodynamic properties of the air/ice/water interface. The present ice ocean models are coarse gridded, and do not contain the small-scale information needed by acoustic propagation and scattering models. Modeling the ice thickness and cover is an important first step; the imbedding of fine-scale structure such as ice roughness into a layered ocean ice model needs to be addressed in the future. At the present time the physics of arctic ice formation and aging is not known adequately to address this topic.

\subsection{Boundary and Volume Scattering}

The ocean acoustic modeling community currently does not have the capability to predict or calculate the 3-D acoustic wavefield scattered from a rough, anelastic, heterogeneous ocean bottom/subbottom or a rough pressure relief surface with associated breaking surface gravity waves (i.e. high sea state and wind friction conditions). In addition, the influence of internal waves on 3-D acoustic propagation is not included in available ocean or acoustic propagation models (50$1000 \mathrm{~Hz}$ ). Ewart (APL University of Washington) does have 2-D numerical models which calculate the acoustic wavefield scattered from the ocean surface and the statistical characteristic of the wavefield propagating through an internal wavefield. He is expanding these models to 3-D. 
The modeling of long range acoustic reverberation was thought to be possible if full elastic 3-D wavefield finite element or finite difference scattering codes (which are slow and limited by the memory capacity of available computers and consequently range limited) were embedded in faster propagation codes such as 3-D PE, coupled mode or ray trace codes which calculate the acoustic wavefield propagating through the water column. The 3-D finite element or finite difference codes would be used to calculate the scattered wave field only when surface or bottom/subbottom interactions occurred.

The calculation of the bottom/subbottom scattered acoustic wavefields requires either a statistical or deterministic characterization of the media. The statistical and spatial scales which are important to the scatter of acoustic energy from the 3-D ocean bottom interface are determined by the acoustic frequency, transmitter and receiver beam patterns and ranges from the scattering entity. It was noted that bottom/subbottom characterization techniques used by the geology and geophysics community do not adequately cover the spatial scales between one and one hundred meters. This roughness scale is critical to acoustic scattering from the bottom/subbottom for the 15 to $1500 \mathrm{~Hz}$ acoustic frequencies band. Orr mentioned that this issue had been discussed at the Terrains Conference which had been held in April 1988. Documentation outlining that meeting is in preparation.

The 3-D calculation of acoustic scattering by surface gravity waves requires surface gravity wave directional spectra as a function of the local and non-local surface gravity wavefield as well as local wind friction. It was noted that the oceanography community can not measure either the deterministic surface wave characteristics or the surface wave spectra over the spatial scales required for the $50-1000 \mathrm{~Hz}$ acoustic frequency band. Experimental techniques to measure surface wave directional spectra over spatial scales relevant to the acoustics community must be developed. 
E.g. for $10-1000 \mathrm{~Hz}$ where $\lambda=150 \mathrm{~m}$ to $1.5 \mathrm{~m}$, we need to get information on surface wavelengths $\lambda_{s}=15 \mathrm{~m}$ to $.5 \mathrm{~m}$.

Internal waves also alter the phase and amplitude of the acoustic wavefield. The effect is frequency dependent and can significantly affect the acoustic wavefield coherence and the gain of large acoustic arrays. It was proposed that the effects of internal waves may have to be initially included in ocean or 3-D propagation models via statistical methods. The small spatial scale of the internal wavefields, the difficulty in obtaining synoptic data over large areas and the intermittent nature of the internal wavefields all contribute to the modeling difficulties.

\subsection{Miscellaneous Issues}

Two issues were repeatedly addressed during the discussions and were still unanswered when the meeting adjourned. The first is what physics must be included in three dimensional acoustic research models? Given the 3-D IFP PE's speed and full wave flavor, other 3-D codes would at first seem superfluous. This, however, is not the case. Ray theory and normal mode codes give physical decompositions of the field which are of great use to tomography, shallow water acoustics, phased array source localization, etc. In addition, finite difference or finite element codes can handle the full elastic problem, as well as rough surfaces scattering. It seems certain that one needs a variety of 3-D codes which can be assembled into embedded or hierarchical 3-D acoustic models.

The second issue concerning acoustic or ocean model intercomparison, is a more difficult one. Matthews' excellent talk pointed out the pitfalls of model intercomparison. The "apples and oranges" situation can easily develop when comparing models. Moreover, by a "judicious" choice of intercomparison examples, one can make one model or another look superior or inferior. For these reasons, people initially backed off considering benchmarking and intercomparison of 3-D acoustic 
models. However, toward the end of the discussions, a feeling that perhaps benchmarking was a good idea won through. Toward that goal, Lee, Siegmann, St. Mary, Schultz, Jacobson, Chiu and Lynch are developing a test data set example using a Harvard "GULFCAST" sound speed field. With this data set, they will test the 3-D PE, mode, and ray programs. They will distribute the test data set to anyone who would like to join in the (very informal at this stage) benchmark comparisons. Anyone wanting the test file can contact them and they will send you the file by tape or computer mail.

\subsection{Concluding Remarks}

These remarks address the issues of accurate prediction of the phase, amplitude and statistical properties of a 3-D acoustic wavefield. They are for the $50-1000 \mathrm{~Hz}$ frequency band and exclude the influence of internal wavefields and smaller scales propagating in the ocean volume. In summary, the workshop brought out the following points:

1. The oceanographic modeling community does not have a quantitative assessment of the accuracy to which the sound velocity and ocean current fields can be predicted using a specified initialization field. Consequently, at the present time the reliability of any acoustic wavefield calculated using ocean model derived sound velocity and current fields is unknown.

2. The ocean acoustic community does not have a quantitative statement concerning the accuracy to which it must be able to predict and measure the phase, amplitude and statistical properties of an acoustic wavefield in the dynamic ocean. A simple statement must be made e.g. measure and predict the phase and amplitude to within $10 \%$ in a 3-D dynamic ocean at a range of $500 \mathrm{~km}$. 
3. The ocean acoustics community has never made a definite field and numerical study to assess the accuracy of existing acoustic models and to determine the minimum uncertainty to which the sound velocity and ocean current input data fields must be known to predict a 3-D acoustic wavefield within specified error.

It should be apparent to the reader that the applied ocean acoustic community will have a much looser definition of uncertainty $\left({ }^{+} \mathrm{x} d \mathrm{~dB}\right)$ than the basic research community $\left({ }^{+} \mathrm{y} d \mathrm{~dB}\right)$. The ocean acoustic basic research community should be attempting to develop the ability to accurately predict a 3-D acoustic wavefield and definitively establish the limits to which such a prediction can be made. It is towards this objective that the oceanography and ocean acoustics communities must attempt to integrate not only their modeling but also their field measurement efforts.

Without establishing the limits to which the ocean acoustic community can predict and measure the phase and amplitude of the acoustic wavefield in the 3-D ocean environment over a broad frequency range, the Navy will not have the necessary knowledge and manpower base available for the design of the next generation of ASW and underwater communication systems. 


\section{Acknowledgements}

We would like to thank our colleagues who attended this meeting for their time and efforts. Special thanks go to Mr. Bob Willems for taking care of the logistics, Ms. Evelyn Lott for clerical assistance, and Ms. Laurel Moore for her secretarial work. Dr. Marshall Orr's (ONR, Code 1125OA) initiation and sponsorship of this workshop are deeply appreciated. We are also grateful to Dr. Marshall Orr as well as Dr. Chris Mooers for their comments and editing of this report. This workshop was jointly coordinated by Woods Hole Oceanographic Institution and the Institute for Naval Oceanography. This work was partially supported by ONR Contract No. N00014-88-K-0363. 


\section{Appendix A: List of Attendees}

Dr. Ralph Baer, NRL, Washington, DC 20375-5000

Dr. Marvin A. Blizzard, ONR, Code 1125AO, 800 No. Quincy St., Arlington, VA 22217-5000

Dr. Homer Bucker, NOSC, San Diego, CA 92152

Mr. Ed Chaika, NORDA, Stennis Space Center, MS 39529

Mr. Ted Charrette, MIT, 77 Massachusetts Ave., MA 02139

Mr. Dave Chester, MIT, 77 Massachusetts Ave., MA 02139

Dr. Stan Chin-Bing, NORDA, Stennis Space Center, MS 39529

Dr. Ching-Sang Chiu, NPGS, Monterey, CA 93943

Dr. Mike Collins, NORDA, Stennis Space Center, MS 39529

Dr. Orest Diachok, NRL, Washington, DC 20375-5000

Mr. Martin Dougherty, WHOI, Geol. \& Geophs. Dept., Woods Hole, MA 02543

Ms. Laura Ehret, INO, Stennis Space Center, MS 39529

Mr. Bob Evans, NOO, Stennis Space Center, MS 39529

Dr. Richard Evans, SYNTEC Eng., 1 Denison Ave., Suite 202, Mystic, CT 06355-2709

Dr. Scott Glenn, Harvard Univ., Dept. of Earth \& Planetary Sci., Pierce Hall, 29 Oxford St., Cambridge, MA 02138

Dr. Martha Head, NORDA, Stennis Space Center, MS 39529 
Dr. Ding Lee, NUSC, Code 3122, New London, CT 06320

Dr. Jim Lynch, WHOI, Applied Ocean Physics \& Eng. Dept., Woods Hole, MA 02543

Dr. Jim Matthews, NORDA, Stennis Space Center, MS 39529

Prof. John McCoy, Catholic University, Dept. of Civil Eng., Washington, DC 20064

Dr. James Mercer, APL, Univ. of Washington, Seattle, WA 98105

Dr. Chris Mooers, INO, Stennis Space Center, MS 39529

Prof. Joe Murphy, University of New Orleans, Dept. of Physics, Lakefron, New Orleans, LA 70148

Dr. Marshall Orr, ONR, Code 11250 A, 800 No. Quincy St., Arlington, VA 22217-5000

Dr. John Perkins, NRL, Washington, DC 20375-5000

Prof. Donald F. St. Mary, Univ. of Massachusetts, Amherst, MA

CDR Manny Salinas, ONR, Code 12, 800 No. Quincy St., Arlington, VA 22217-5000

Prof. Bill Siegmann, Rensselaer Polytechnic Institute, Dept. of Math. Sci., Troy, NY 12181

Prof. Frederick Tappert, Univ. of Miami, RSMAS, 4600 Rickenbacker Cswy., Miami, FL 33149

Prof. Herbert Uberall, Catholic University, Dept. of Physics, Washington, DC 20064

Dr. Henry Weinberg, SYNTEC Eng., 1 Denison Ave., Suite 202, Mystic, CT 06355-2709

Mr. Bob Willems, INO, Stennis Space Center, MS 39529 


\section{Appendix B: List of Presentations}

1. Harvard Open Ocean Model, Scott Glenn

2. A 3-Dimensional model - FOR3D, Ding Lee

3. 3-D ray acoustics, Jim Lynch

4. 3-D coupled modes, Ching-Sang Chiu

5. Wide-area rapid acoustic prediction (WRAP), John Perkins

6. Model Evaluations, Jim Matthews

7. Gaussian Beams, Homer Bucker

8. 3-D nonreciprocity, James Mercer

9. 3-D stepwise coupled modes/triangulation, Richard Evans

10. PE modeling, Frederick Tappert

11. Finite element model for ocean acoustic propagation, Joe Murphy

12. Phase space marching and filtering, John McCoy

13. Ocean bottom absorption and shear effects and 3-D effects in coupled mode propagation theory, Herbert Uberall

14. PE modeling with surface loss, Martha Head

15. Seismic/acoustic finite difference modeling, Martin Dougherty

16. Pseudospectral Modeling, Ted Charrette 
17. Vertical modes and horizontal rays, Henry Weinberg

18. Three-dimensional PE and ray tracing modeling, Michael D. Collins

19. Bi-static reverberation acquired from shot data, Henry Weinberg 
Attn: Stella Sanchez-Wade

Documents Section

Scripps Institution of Oceanography

Library, Mail Code C-075C

La Jolla, CA 92093

Hancock Library of Biology \& Oceanography

Alan Hancock Laboratory

University of Southern California

University Park

Los Angeles, CA 90089-0371

Gifts \& Exchanges

Library

Bedford Institute of Oceanography

P.O. Box 1006

Dartmouth, NS, B2Y 4A2, CANADA

Office of the International

Ice Patrol

c/o Coast Guard R \& D Center

Avery Point

Groton, CT 06340

Library

Physical Oceanographic Laboratory

Nova University

8000 N. Ocean Drive

Dania, FL 33304

NOAA/NESDIS Miami Library Center 4301 Rickenbacker Causeway

Miami, FL 33149

Library

Skidaway Institute of Oceanography

P.O. Box 13687

Savannah, GA 31416

Institute of Geophysics

University of Hawaii

Library Room 252

2525 Correa Road

Honolulu, HI 96822

Library

Chesapeake Bay Institute

4800 Atwell Road

Shady Side, MD 20876

MIT Libraries

Serial Journal Room 14E-210

Cambridge, MA 02139
Director, Ralph M. Parsons Laboratory

Room 48-311

MIT

Cambridge, MA 02139

Marine Resources Information Center

Building E38-320

MIT

Cambridge, MA 02139

Library

Lamont-Doherty Geological Observatory

Colombia University

Palisades, NY 10964

Library

Serials Department

Oregon State University

Corvallis, OR 97331

Pell Marine Science Library

University of Rhode Island

Narragansett Bay Campus

Narragansett, RI 02882

Working Collection

Texas A\&M University

Dept. of Oceanography

College Station, TX 77843

Library

Virginia Institute of Marine Science

Gloucester Point, VA 23062

Fisheries-Oceanography Library

151 Oceanography Teaching Bldg.

University of Washington

Seattle, WA 98195

Library

R.S.M.A.S.

University of Miami

4600 Rickenbacker Causeway

Miami, FL 33149

Maury Oceanographic Library

Naval Oceanographic Office

Bay St. Louis

NSTL, MS 39522-5001

Marine Sciences Collection

Mayaguez Campus Library

University of Puerto Rico

Mayagues, Puerto Rico 00708 


\begin{tabular}{|c|c|c|c|}
\hline $\begin{array}{l}\text { REPORT DOCUMENTATION } \\
\text { PAGE }\end{array}$ & $\begin{array}{l}\text { 1. REPORT NO. } \\
\text { WHOI-89-16 }\end{array}$ & 2. & 3. Recipient's Accession No. \\
\hline \multirow{2}{*}{\multicolumn{3}{|c|}{$\begin{array}{l}\text { 4. Title and Subtitle } \\
\text { A Report on the 3-D Acoustic Working Group Meeting at Long Beach, MS, July 7-8, } \\
1988\end{array}$}} & $\begin{array}{l}\text { 5. Report Date } \\
\text { June } 1989\end{array}$ \\
\hline & & & 6. \\
\hline \multicolumn{3}{|c|}{$\begin{array}{l}\text { 7. Author(s) } \\
\text { James F. Lynch, Ching-Sang Chiu }\end{array}$} & $\begin{array}{l}\text { 8. Performing Organization Rept. No. } \\
\text { WHOI-89-16 }\end{array}$ \\
\hline \multirow{2}{*}{\multicolumn{3}{|c|}{$\begin{array}{l}\text { 9. Performing Organization Name and Address } \\
\text { The Woods Hole Oceanographic Institution } \\
\text { Woods Hole, Massachusetts } 02543\end{array}$}} & 10. Project/Task/Work Unit No. \\
\hline & & & $\begin{array}{l}\text { 11. Contract(C) or Grant(G) No. } \\
\text { (C) N00014-88-K-0363 } \\
\text { (G) }\end{array}$ \\
\hline \multirow{3}{*}{\multicolumn{3}{|c|}{$\begin{array}{l}\text { 12. Sponsoring Organization Name and Address } \\
\text { The Office of Naval Research }\end{array}$}} & 13. Type of Report \& Period Covered \\
\hline & & & Technical Report \\
\hline & & & 14. \\
\hline
\end{tabular}

\section{Supplementary Notes}

This report should be cited as: Woods Hole Oceanog. Inst. Tech. Rept., WHOI-89-16.

16. Abstract (Limit: 200 words)

At the request of ONR Code 1125OA, the Woods Hole Oceanographic Institution (Dr. James F. Lynch) convened a workshop to bring together a group of acoustic and ocean modelers to review and discuss

1. the state of development and the need for three-dimensional numerical acoustic research propagation and scattering models;

2. The interfacing of acoustic models with available oceanographic data and ocean model outputs.

The workshop was hosted by the Institute for Naval Oceanography (Dr. Ching-Sang Chiu) at Long Beach, MS on July 7-8, 1988. This report summarizes the research presentations and the recommendations made by the group. The workshop was an initial attempt to promote the interaction between the ocean and acoustic modeling communities. This interaction between the communities is essential to the development of truly interactive basic research acoustic and ocean models. We anticipate more workshops of such nature to be held in the future. The findings and recommendations generated by these workshops are expected to have a strong impact on the direction of future three-dimensional modeling research in both acoustics and oceanography.

17. Document Analysis a. Descriptors

1. 3-D Acoustic Propagation Model

2. Ocean Numerical Modeling

3. Ocean Model/Acoustic Model Interface

b. Identifiers/Open-Ended Terms

c. COSATI Field/Group

18. Availability Statement

Approved for publication; distribution unlimited.

\begin{tabular}{|c|c|}
\hline $\begin{array}{c}\text { 19. Security Class (This Report) } \\
\text { UNCLASSIFIED }\end{array}$ & $\begin{array}{c}\text { 21. No. of Pages } \\
25\end{array}$ \\
\hline 20. Security Class (This Page) & 22. Price \\
\hline
\end{tabular}

